\title{
Survival analysis of patients with metastatic bone disease administered anti-resorptive agents
}

Tomoko HIGUCHI $(1,2)$, Yoshihiko SOGA (1), Akira SASAKI (2)

1. Division of Hospital Dentistry, Okayama University Hospital, Okayama, Japan

2. Department of Oral and Maxillofacial Surgery and Biopathology, Okayama University Graduate School of Medicine OKAYAMA

Dentistry and Pharmaceutical Sciences, Okayama, Japan

Backgrounds Anti-resorptive agents are administered during the overall management of patients with metastatic bone disease to delay and prevent skeletal-related events and other side effects of anti-resorptive agent-related osteonecrosis of the jaw (ARONJ).

Case 1. Anti-resorptive agents started before dental examination.

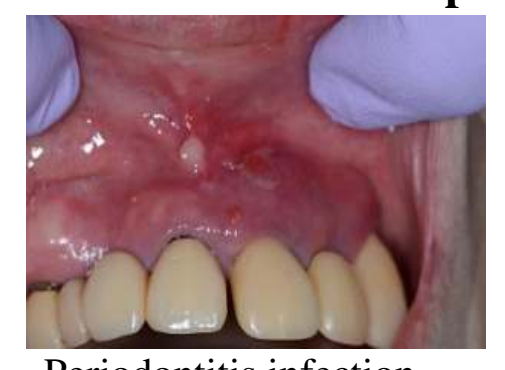

Periodontitis infection

$\rightarrow 21$ Extraction

If the patient was to undergo an examination before being administerd anti-resorptive
agents, would the effects of ARONJ that reduce quality of life be lessened?

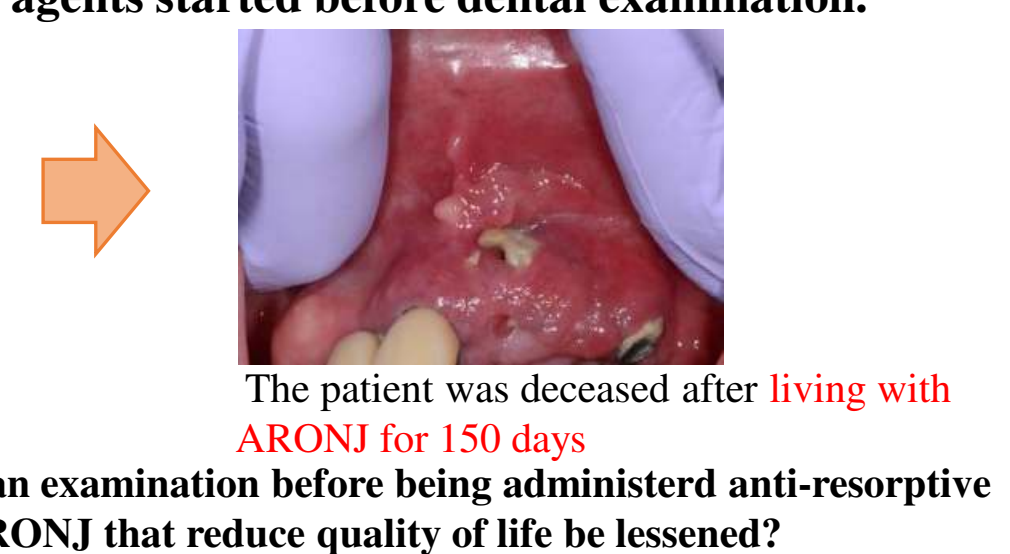

Case 2. The patient was deceased soon after pre-treatment dental examination.

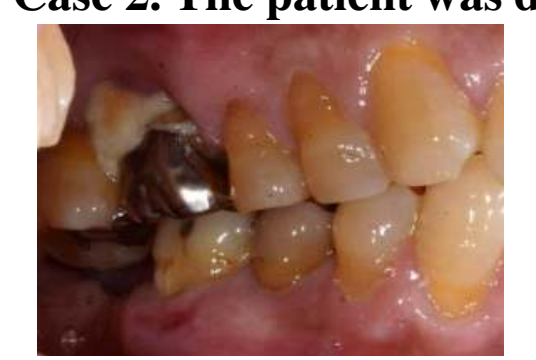

Periodontitis infection $\rightarrow 1646$ Extraction life have been better?

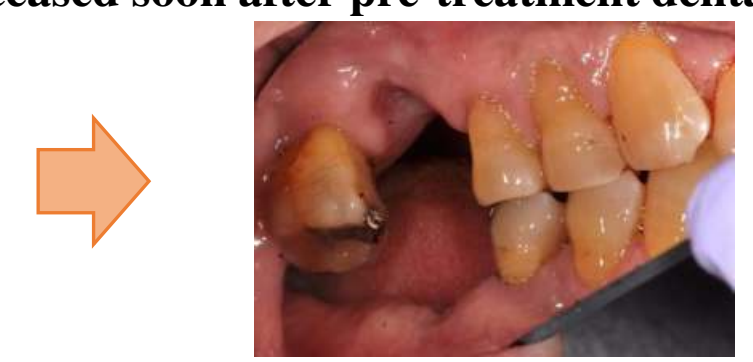

The patients could not bite on the right side and he passed away 10 days after the extraction.

During life, performing dental treatment and oral care is imperative in patients irrespective of ARONJ.

Objectives This retrospective study is aimed to evaluate the overall survival rates of patients with metastatic bone disease in our hospital.

\section{Participants and Methods}

Study Design

This was a single-center, retrospective, observational study.

\section{Participants}

Patients who were administered anti-resorptive agents for metastatic bone disease from April 2012 to March 2015 [n=270, M:114, F:156, 63 (26-90)y]

Methods

The Kaplan-Meier analysis and log-rank test were used to estimate survival rates of all participants, including those administered zoledronic acid, or denosumab and those with a primary tumor. Statistical analysis was performed by a statistical software; Stata14 (StataCorp LLC, TX, USA).

\begin{tabular}{cc}
\hline Table 1. Anti-resorptive agents & \\
\hline Anti-resorptive agents & $\mathbf{n}$ \\
\hline $\begin{array}{c}\text { Zoledronic acid and } \\
\text { Replacement of Zoledronic acid } \\
\text { with denosumab }\end{array}$ & 110 \\
denosmab & 160 \\
\hline Total & 270 \\
\hline
\end{tabular}

Zoledronic acid or denosumab administration Zoledronic acid was administered at a dose of $4 \mathrm{mg}$ every $3-6$ weeks over 15 minutes by intravenous injection, and denosub was administered at a dose of $120 \mathrm{mg}$ every $4-5$ weeks, by subcutaneous injection. Zoledronic acid dose adjustment was carried out base on the patient's calcium level and/or renal function.

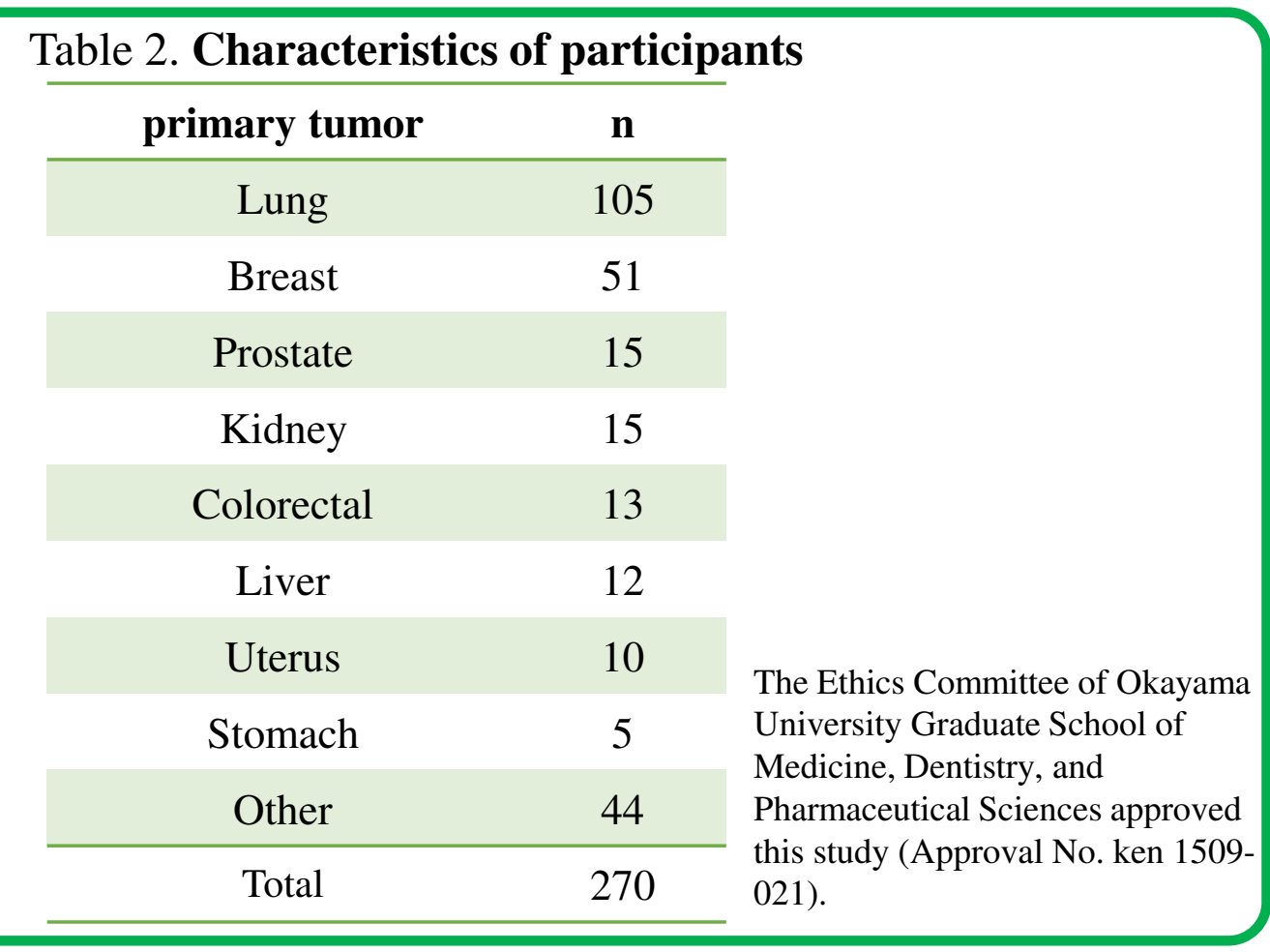

Results

The Kaplan-Meier survival estimates and survival rates from starting administered anti-resorptive agents in metastatic bone disease.

1) All participants

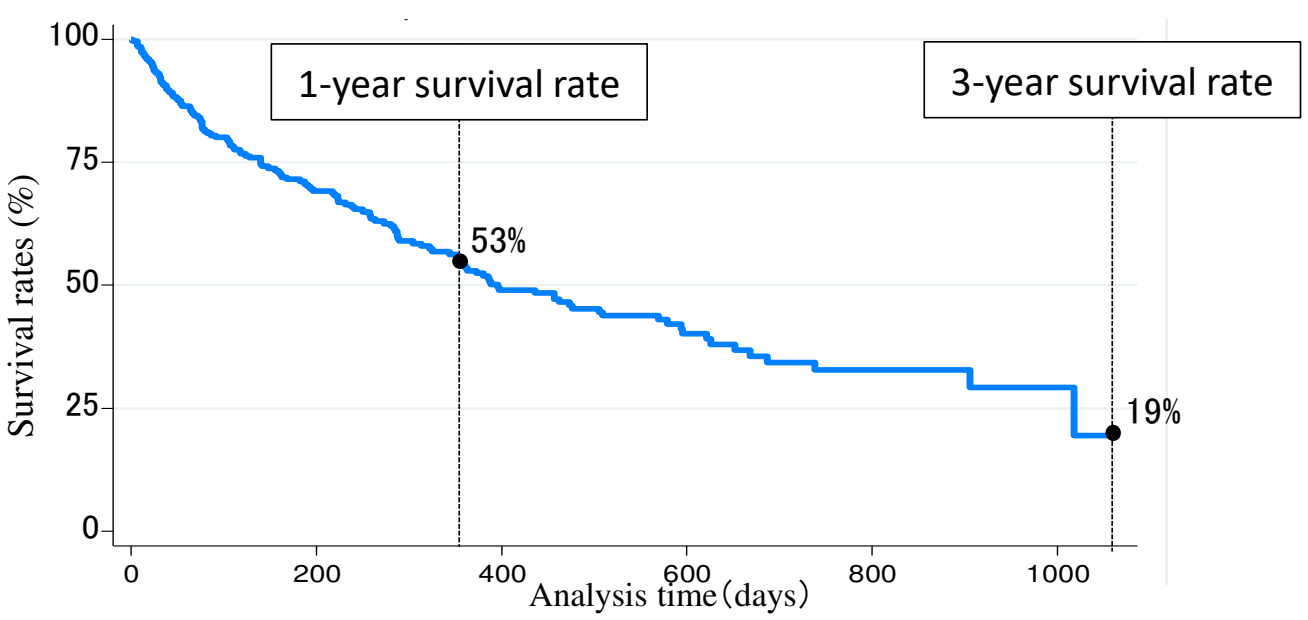

Figure 1. The Kaplan-Meier survival estimates of all participants

3) Primary tumor

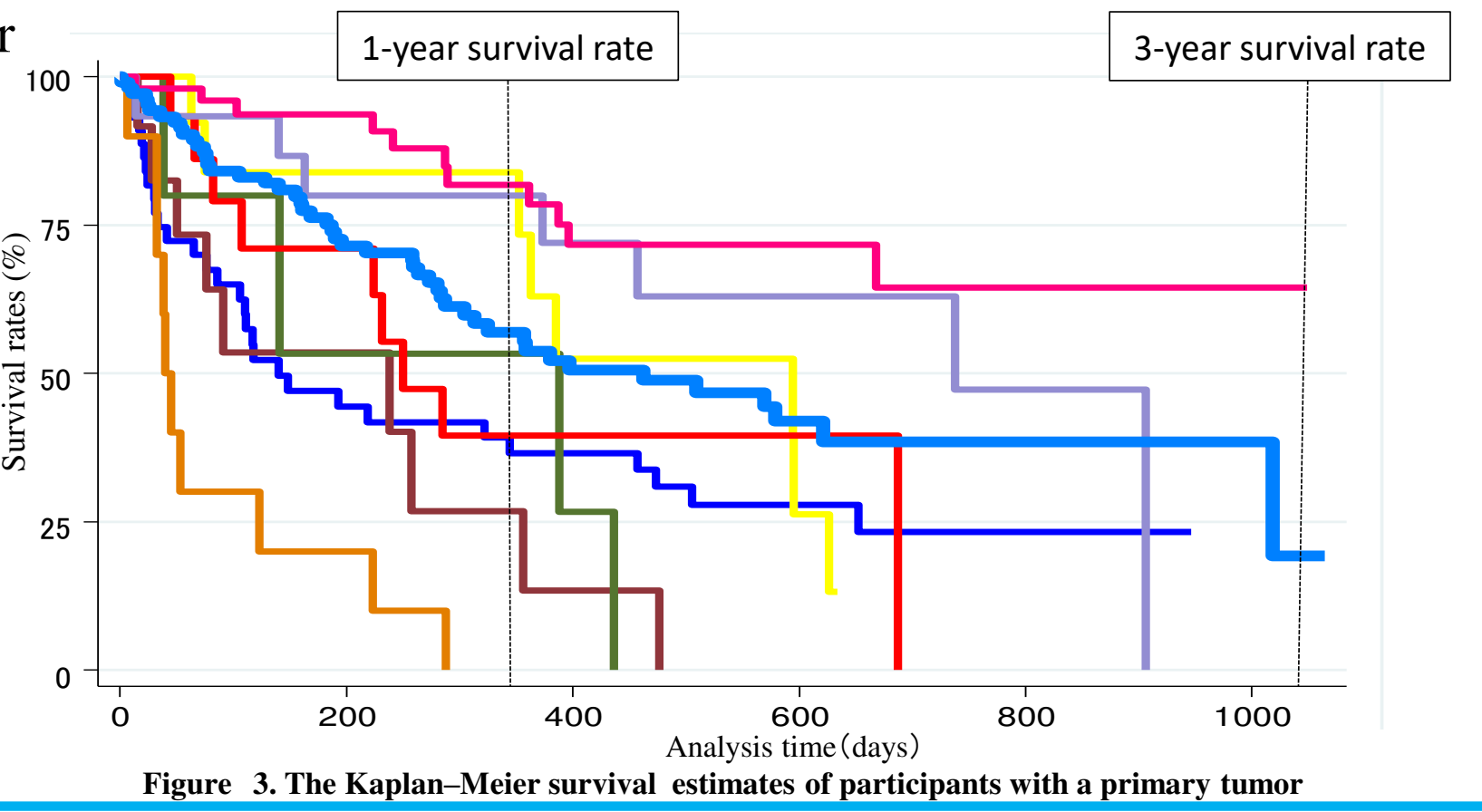

2) Zoledronic acid or denosumab

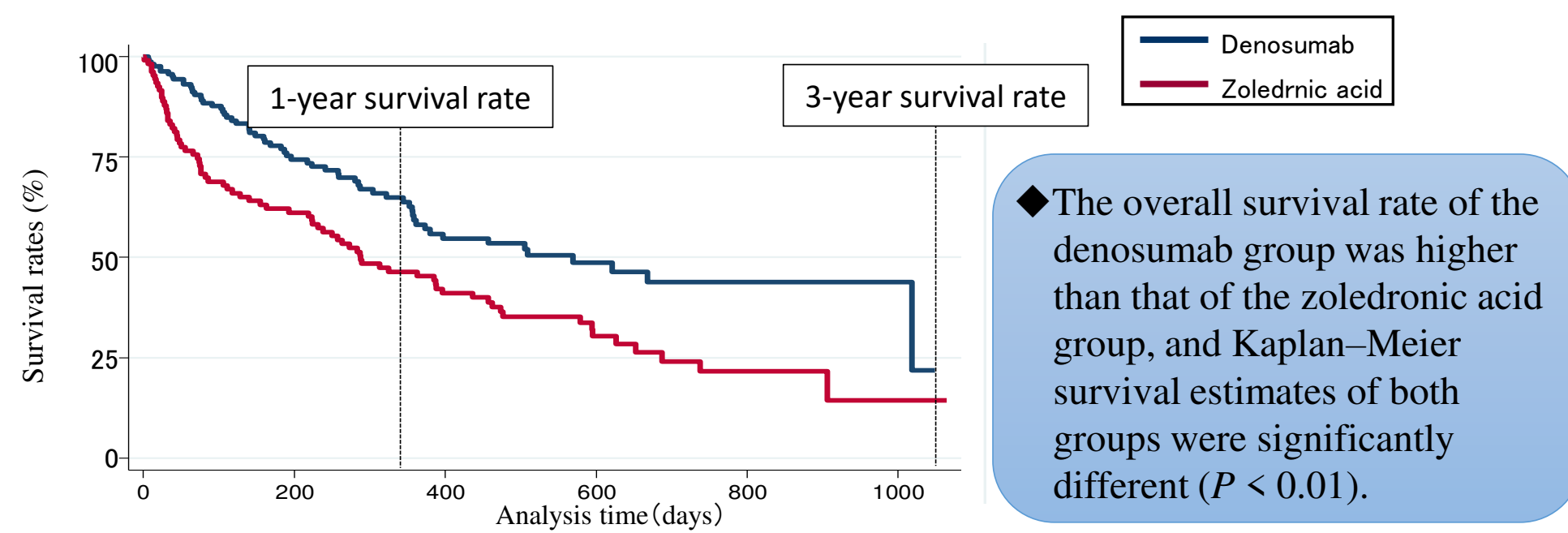

Figure 2. The Kaplan-Meier survival estimates of participants given zoledronic acid or denosumab

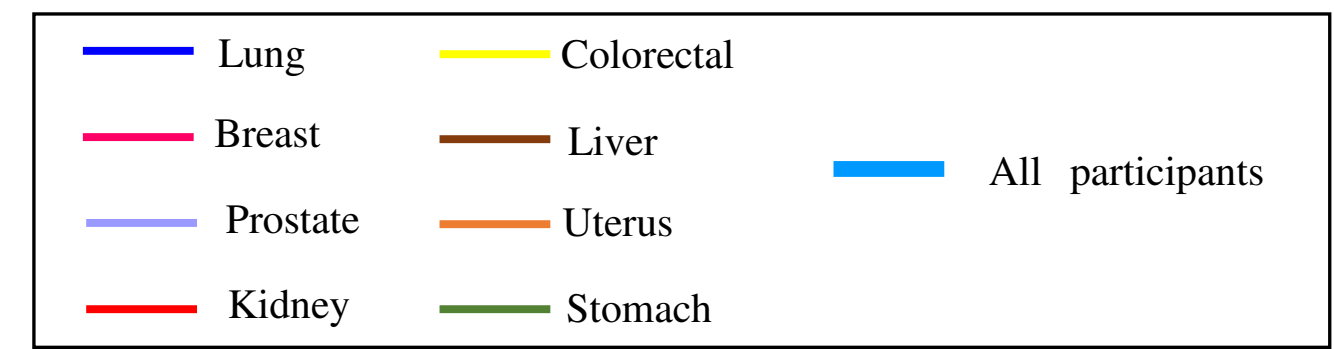

The overall survival rate of the breast cancer group was higher than that of the group of other primary tumors, and Kaplan-Meier survival estimates of both groups were significantly different $(P<0.01)$.

Discussions - Considering the survival rates, treatment plans including the removal of dental infection should be constructed.

- It is necessary to predict long-term prognosis and oral management for breast cancer patients who received denosumab as a treatment in particular, because they have significantly higher survival rates than other patients. 